\title{
Fluorescent-Based Quantitative Measurements of Signal Transduction in Single Cells
}

\author{
Pelet, Serge ; Peter, Matthias
}

\begin{abstract}
Budding yeast (Saccharomyces cerevisiae) has been widely used as a model system to study fundamental biological processes. Genetic and biochemical approaches have allowed in the last decades to uncover the key components involved in many signaling pathways. Generally, most techniques measure the average behavior of a population of cells, and thus missed important cell-to-cell variations. With the recent progress with fluorescent proteins, new avenues have been opened to quantitatively study the dynamics of signaling in single living cells. In this chapter, we describe several techniques based on fluorescence measurements to quantify the activation of biological pathways. Flow cytometry allows for rapid quantification of the total fluorescence of a large number of single cells. In contrast, microscopy offers a lower throughput but allows to follow with a high temporal resolution the localization of proteins at sub-cellular resolution. Finally, advanced functional imaging techniques such as FRET and FCS offer the possibility to directly visualize the formation of protein complexes or to quantify the activity of proteins in vivo. Together these techniques present powerful new approaches to study cellular signaling and will greatly increase our understanding of the regulation of signaling networks in budding yeast and beyond.
\end{abstract}

DOI: https://doi.org/10.1007/978-1-4419-6766-4_17

Posted at the Zurich Open Repository and Archive, University of Zurich

ZORA URL: https://doi.org/10.5167/uzh-79446

Book Section

Originally published at:

Pelet, Serge; Peter, Matthias (2011). Fluorescent-Based Quantitative Measurements of Signal Transduction in Single Cells. In: Koeppl, Heinz; Densmore, Douglas; Setti, Gianluca; di Bernardo, Mario. Design and Analysis of Biomolecular Circuits: Engineering Approaches to Systems and Synthetic Biology. New York, USA: Springer, 369-394.

DOI: https://doi.org/10.1007/978-1-4419-6766-4_17 


\title{
Chapter 6.3
}

\section{FLUORESCENT-BASED QUANTITATIVE MEASUREMENTS OF SIGNAL TRANSDUCTION IN SINGLE CELLS}

\author{
Serge Pelet and Matthias Peter \\ Institute of Biochemistry, Department of Biology, ETH Zürich
}

\begin{abstract}
Budding yeast (Saccharomyces cerevisiae) has been widely used as a model system to study fundamental biological processes. Genetic and biochemical approaches have allowed in the last decades to uncover the key components involved in many signaling pathways. Generally, most techniques measure the average behavior of a population of cells, and thus missed important cell-tocell variations. With the recent progress with fluorescent proteins, new avenues have been opened to quantitatively study the dynamics of signaling in single living cells. In this chapter, we describe several techniques based on fluorescence measurements to quantify the activation of biological pathways. Flow cytometry allows for rapid quantification of the total fluorescence of a large number of single cells. In contrast, microscopy offers a lower throughput but allows to follow with a high temporal resolution the localization of proteins at sub-cellular resolution. Finally, advanced functional imaging techniques such as FRET and FCS offer the possibility to directly visualize the formation of protein complexes or to quantify the activity of proteins in vivo. Together these techniques present powerful new approaches to study cellular signaling and will greatly increase our understanding of the regulation of signaling networks in budding yeast and beyond.
\end{abstract}

Key words: Cellular signaling, Fluorescent proteins, Microscopy, Flow cytometry, FRET, FCS.

\section{INTRODUCTION}

Cells have engineered elaborate capacities to sense their environment in order to detect nutrient sources, hormones or stress. These extra-cellular cues 
are often sensed by membrane-associated receptors, which transduce this information inside the cell via signaling cascades. These highly interconnected biochemical pathways can integrate multiple inputs to deliver the appropriate response such as proliferation, differentiation, adaptation or apoptosis. These various cellular fates are generally controlled by the activation of key signaling molecules and/or the activation of a specific transcriptional expression program.

To better understand these signaling networks and to build predictive mathematical models, it is essential to gather quantitative measurements at different steps along the information transduction cascade. For many decades, biologists have optimized standard biological methods such as western blotting and mass spectrometry to deliver more accurate and quantitative estimations of protein levels and modifications. However, their requirements for large quantities of material generally limit these techniques to the measurements of the average response of a population to a stimulus.

\subsection{Single cell analysis}

Since the seminal work from Ferrel and Machleder performed on single maturing oocytes ${ }^{1}$, it has been realized that population averaged measurements can prevent the discovery of more complex regulatory mechanisms. As schematically represented in Figure 1, the output of a pathway can increase gradually with stimulus or display an all-or-none Stimulus
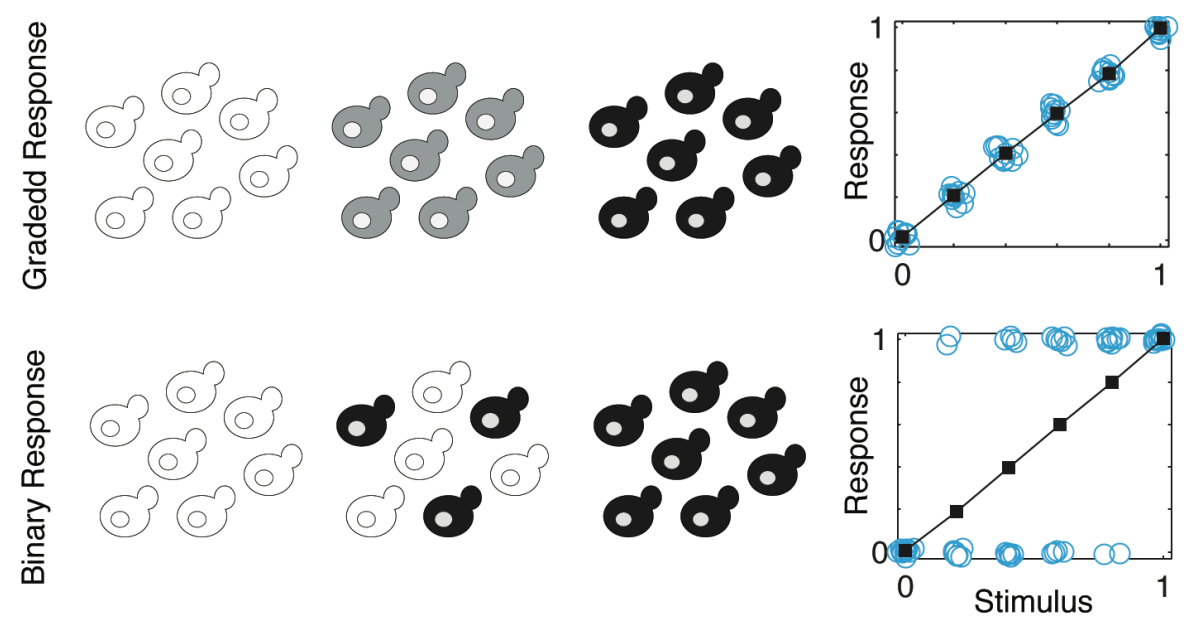

Figure 1. Schematic difference between a graded and binary response to an increasing stimulus. The graphs show how different single cell behaviours (o) can lead to an identical population measurement (घ). 
output pattern. However, due to stochastic differences between individual cells, the population averaged measurements can be identical in both situations. Only measurements performed at the single cell level can reveal this fundamental difference in signal transduction.

In the case of the maturing oocyte ${ }^{1}$, the averaged population response displays an apparent Hill coefficient of one, which is typical for a graded response. Fitting of the single cell measurements results in a Hill coefficient of 42 , which describe a highly switch-like activation. The discovery of this ultra-sensitive response allowed the authors to postulate the presence of a positive feedforward loop, acting at the transcriptional level. Inhibition of the protein production by addition of cycloheximide renders the output of the signaling cascade more graded.

This example of the ability of single cell measurements to uncover hidden regulatory mechanisms is being applied to a wide variety of signal transduction pathways ${ }^{2,3}$. Signaling cascades often include such feedback or feedforward loops to exquisitely control the output of the pathway or generate ultra-sensitivity of the cellular response. Moreover the dynamics of signal transduction can be best studied at the single cell level since many informative behaviors, such as oscillations or activity bursts, could be averaged out in population measurements ${ }^{4,5}$.

\subsubsection{Cellular noise}

As mentioned in the previous section, the different outcomes measured at the output of a signaling cascade can arise from small stochastic differences between individual cells ${ }^{6}$. Therefore single cell experiments have been designed to study the source of cellular noise. Using a set of yellow and cyan fluorescent reporters, which are under the control of the same promoter, Elowitz and co-workers ${ }^{7}$ defined the concept of intrinsic and extrinsic noise.

As sketched in Figure 2, if all cells were well-stirred chemical reactors with an identical number of internal components, one would expect them to produce the same amount of cyan and yellow fluorescent proteins. However, it is well known that each cell is different from the others with a unique composition of proteins (number of polymerases and ribosomes), even within a clonal population ${ }^{7,8}$. Under these conditions, one would expect each individual cell to have various abilities to express proteins. However, in a given cell, the cyan and yellow fluorescent proteins should be expressed at the same level. This cell-to-cell variation is defined as the extrinsic noise. There is an additional component of noise that will uncouple the cyan and yellow expression levels. Due to a limited number of components, the expression of each individual gene can become more stochastic. If there are 


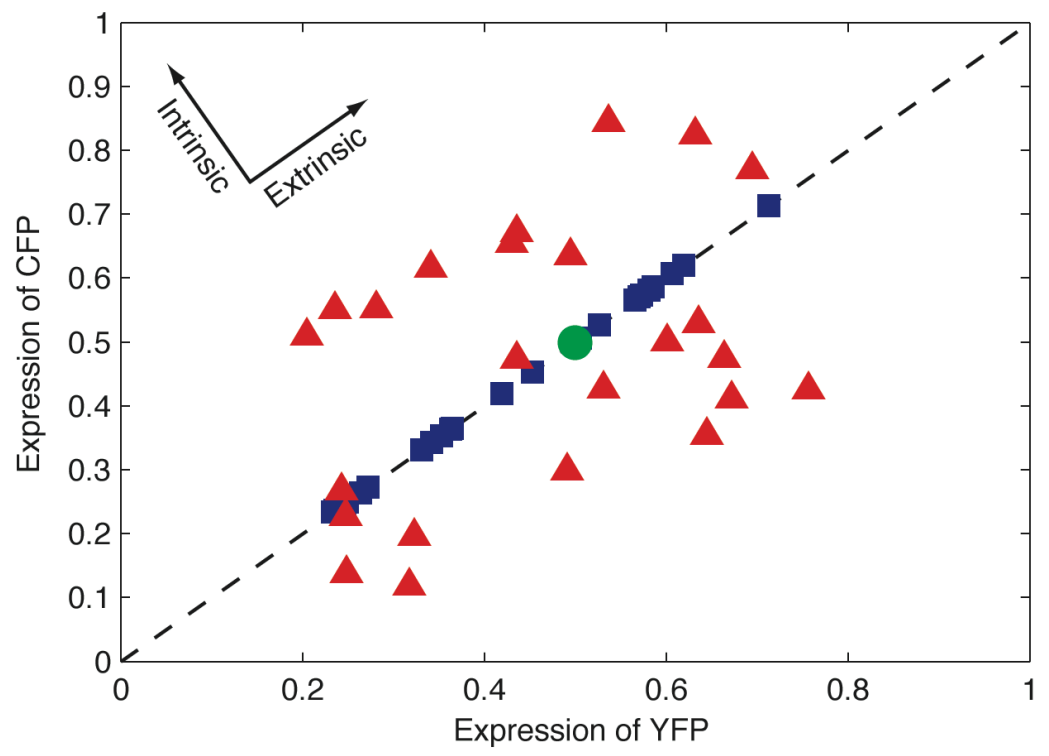

Figure 2. Representation of intrisic and extrinsic noise in a biological system. In absence of noise, the expression of yellow (YFP) and cyan (CFP) reporter proteins under the control of the same promoter would be identical in all cells $(\bullet)$. In the sole presence of extrinsic noise, the expression of YFP and CFP would be highly correlated ( $\mathbf{})$. In the presence of stochastic variations in each cell, YFP and CFP can be expressed at different levels in the same cell $(\mathbf{\Delta})$. for instance only a small number of transcription factors that can promote the expression of the cyan and yellow reporters, the final amount of protein produced will depend on the stochastic binding event of the transcription factor to either promoter and will therefore not be identical for both genes. The noise, which results in the variation of the cyan and yellow proteins levels in the same cell, is defined as the intrinsic noise.

The ability to isolate the origin of the cellular noise can help understand the processes that control the signaling dynamics of a given systems. As an example, Colman-Lerner et al. ${ }^{9}$ found a high level of correlation between the expression of a reporter specific for a signaling cascade and the expression of a constitutively expressed gene. This implies that most of the noise in expression comes from the ability of the individual cells to produce proteins (defined as expression capacity). Importantly, this demonstrates that the transduction of the signal occurs in this pathway with a high fidelity although the number of signaling molecules varies from cell to cell.

\subsubsection{Single cell measurements}

In the study from Ferrell and Machleder ${ }^{1}$, the large size of the oocyte allowed them to perform biochemical experiments directly on individual cells. This is unfortunately not the case for the majority of the commonly 
used biological model systems where proteins are found at too low abundance for biochemical investigations. Although recent advances in mass spectroscopy have allowed to reach single cell detection levels for some proteins or metabolites ${ }^{10,11}$, this technique is not yet generally used to analyze signal transduction pathways but might add, in the future, a different approach to analyze the content of single cells. For this chapter, we will focus our discussion on the experimental methods that currently offer single cell resolution which are optical methods such as microscopy and flow cytometry.

Different imaging modalities can be used to visualize cells in a microscope. Absorption or interference methods generate a contrast in the image. However, their sensitivity is limited by the fact that one measures a relative change in the intensity of the transmitted light. On the opposite, fluorescence microscopy offers a much higher sensitivity, since one measures the apparition of a signal over a dark background. Detectors such as photo-multiplier tube or electron-multiplying-CCD have single photon sensitivities. Within the linearity range of the detector, the fluorescence signal will scale linearly with the amount of fluorophore present in the sample. The possibility to image single proteins in living cells has been demonstrated recently ${ }^{12,13}$. Therefore fluorescent microscopy has evolved as the most commonly used imaging modality with high specificity and unmatched sensitivity.

Flow cytometry has been specifically developed to analyze single cells in solution. While the first instruments were based on impedance or absorption measurements, fluorescence rapidly emerged as the preferred detection method, thus enabling the development of fluorescent probes used for both microscopy and flow cytometry.

Table 1. Differences between flow cytometry and microscopy

\begin{tabular}{ccc}
\hline & Microscopy & Flow cytometry \\
\hline Number of cells per & $10^{2}$ & $10^{5}$ \\
measurement & Complex & Simple \\
Data analysis & Yes & No \\
Time-lapse & Sub-cellular resolution & Total cellular intensity \\
Measurement
\end{tabular}

Although both techniques allow the quantification of fluorescent signal emerging from cells, the resulting measurements have different characteristics. The typical number of cells measured by microscopy is on the order of 100 per image, while flow cytometry can easily measure 10'000 cells, thereby generating statistically more significant measurements. Microscopy with time-lapse imaging and subcellular resolutions can offer 
more insight in the sample at the expense of a more complex data analysis. Therefore these two techniques provide different and complementary measurements.

\subsection{Fluorescent proteins}

For many decades chemical dyes were the only contrast method used for fluorescent detection. A large variety of compounds have been synthesized specifically to tag organelles or report on ionic changes in cells. Fluorescently-tagged antibodies have also been extensively used to target fluorescent probes to specific proteins. Unfortunately, these dyes are often toxic for the cell and use of antibody staining requires fixation of the specimen, thereby excluding live cell imaging. The discovery of the green fluorescent protein (GFP) ${ }^{14,15}$ has opened a new area in the field of fluorescent microscopy. It became possible to genetically encode a fluorescent tag for any protein by extending its sequence with the one from GFP. It therefore became possible to visualize, in living cells, the location and dynamic behavior of any protein ${ }^{16}$. As shown in Table 1, a large variety of fluorescent proteins have been engineered ${ }^{17}$. From the original GFP, a number of spectral variants have been created by mutations of only a few residues, such as the cyan and yellow fluorescent proteins. Generation of bright and monomeric red proteins has been more challenging, but many variants are now also available. Codon optimized variants for different organisms have been generated to allow for better expression of the proteins.

Table 2. Commonly used fluorescent proteins

\begin{tabular}{cccl}
\hline Protein & $\lambda_{\text {Excitation }}$ & $\lambda_{\text {Emission }}$ & Other Variants \\
\hline ECFP & 433 & 475 & Cerulean, CyPet, mTFP \\
EGFP & 488 & 507 & Emerald, Azami green \\
EYFP & 514 & 527 & Venus, mCitrine, YPet \\
mCherry & 587 & 610 & mRFP, dsRed, tdTomato \\
\hline
\end{tabular}

There are however a few drawbacks associated with the use of fluorescent proteins. Firstly, as with any other protein tags, the addition of the fluorescent protein ( $\sim 240$ amino acids) to the native sequence of a protein can sometimes impair its function and thereby lead to a mislocalization of the protein. Secondly, the fluorescence of the protein can be affected by its environment in the cell. Although the chromophore is shielded by the $\beta$-barrel structure of the protein, cations often lead to changes in the brightness of the proteins. This can also be taken advantage of as, for instance, a $\mathrm{pH}$-sensitive fluorescent protein has been generated ${ }^{18}$. The photo-stability of the fluorophore can also be an issue, which can lead to degradation of the signal after extended illuminations. Researchers have also 
put that drawback to use by studying the recovery of a fluorescence signal after photo-bleaching (FRAP) ${ }^{19,20}$. This method has allowed to uncover very fast protein dynamics and the possible applications of this technique have been extended by the engineering of photo-activable (PA-GFP) $)^{21}$ or photoswitchable (Kaede) ${ }^{22}$ fluorescent proteins.

Finally, the slow maturation time of GFP can result in some experimental artifacts. The fluorescent protein is expressed and folded rapidly, but the apparition of the fluorescence is delayed by roughly 30 to 45 minutes due to a slow oxidation reaction which needs to take place at the core of the protein to form the chromophore ${ }^{14}$. Despite these few technical issues, fluorescent proteins have revolutionized the bio-imaging field in the last two decades. Engineering of newer, brighter and more stable version will allow to image less abundant proteins for extended periods of time.

\subsection{Budding yeast as a model organism}

Saccharomyces cerevisiae is a small single cell, eukaryotic organism. It is characterized by an asymmetric cell division, which coined its common name: budding yeast. The daughter cell grows as a bud from the mother cell and separates after nuclear division and cytokinesis. Its genome has been sequenced more than 10 years ago ${ }^{23}$ and to date approximately $75 \%$ of the open reading frames have been assigned a function. The biochemical tools developed in yeast to manipulate its genome offer a great advantage over other model systems. It is possible to modify genes at their endogenous genomic locus and therefore express proteins tagged with GFP at their physiological level. Since over-expression of proteins often perturbs the dynamics of signal transduction, retaining the native conditions of a signaling cascade avoids artifacts.

As a unicellular fungus, budding yeast shares many properties common to both plants and animals. Due to the evolutionary conservation of cellular pathways, it has been widely used as model system to study cell cycle regulation, metabolism and signal transduction. Mechanisms used in yeast to transduce information from the exterior to the interior of the cells are also present in higher eukaryotes. As an example, the mitogen activated protein kinase (MAPK) pathway, which consists of a module of three kinases that activate each other is conserved from yeasts to mammals ${ }^{24}$. Misregulation of MAPK signal transduction in mammalians cells has been implicated in multiple diseases such as cancer and inflammation. These pathways are therefore considered as potential drug targets. Although they serve different functions, the general architecture of MAPK pathways is conserved. 


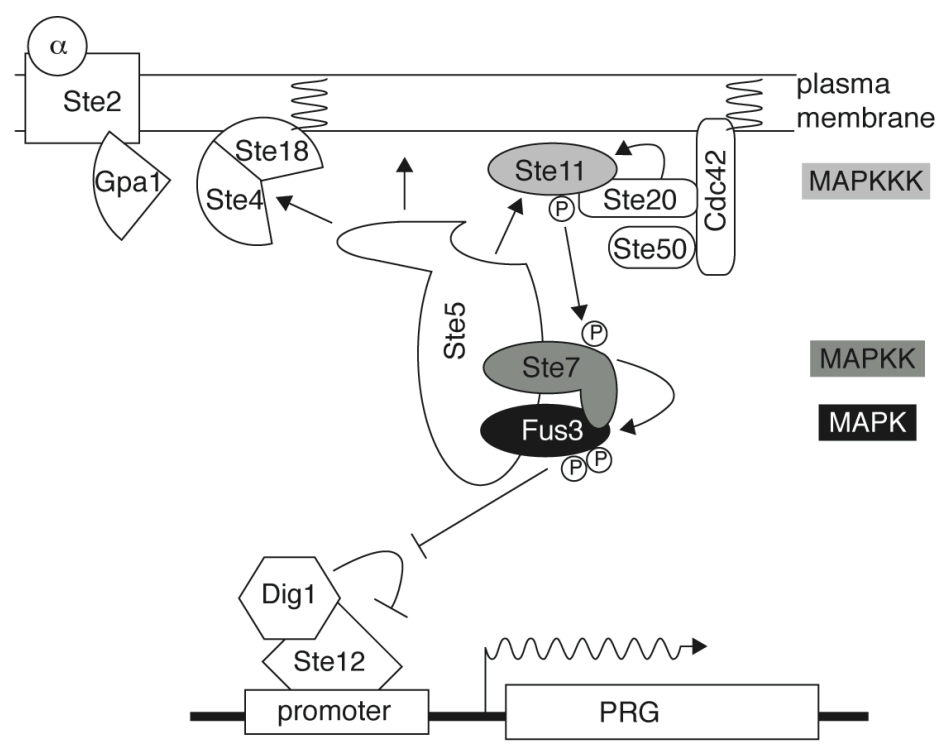

Figure 3. Schematic drawing of the pheromone signalling pathway. Relocation of the scaffold protein Ste $5 p$ to the plasma membrane results in the activation of the MAPK Fus3p, which can in turn promote expression of pheromone responsive genes (PRG).

Therefore considerable work has been invested into understanding the regulation of these pathways in the simplified setting offered by yeast.

Possibly the best understood signal transduction cascade is the MAPK mating pathway in budding yeast $\mathrm{t}^{25,26}$. Decades of thorough genetic and biochemical experiments have allowed to identify the components and architecture of this signaling pathway. The challenge is now to understand how these proteins work together to generate a faithful and robust output in response to the stimulus. Quantitative single cell measurements have been widely employed to achieve this task. Below, we will illustrate each technique presented by an example of their application to the yeast mating pathway.

Haploid yeast (mat-a or mat- $\alpha$ ) sense mating pheromones ( $\alpha$ - or a-factor) secreted by a cell of opposite mating type. As depicted in Figure 3, the pheromone binds to a G-protein coupled receptor (Ste2p) which triggers dissociation of the trimeric G-protein. The free G $\beta \gamma$-subunit (Ste4p/Ste18p) recruits the scaffold protein Ste $5 p$ to the plasma membrane. This scaffold protein has binding sites for all three kinases of the MAPK cascades: Fus3p, the MAP kinase; Ste 7p, the MAP kinase kinase; Ste11p, the MAP kinase kinase kinase. This latter protein is the most up-stream components of the cascade and resides pre-activated at the plasma membrane. Recruitment of Ste $5 p$ to the membrane brings Ste $7 p$ in close proximity of Ste11p, and results in the activation of the pathway by phosphorylation of Ste7p which, in turn, phosphorylates Fus $3 p^{27}$. Activated Fus3p orchestrates the mating 
response by phosphorylating multiple targets, among them Dig1p, which represses transcription of pheromone response genes by inhibiting the transcription factor Ste12p. All these events will result in the arrest of the cell cycle in G1 and the extension of a polarized mating projection towards the source of pheromone.

\section{FLOW CYTOMETRY}

Fluorescence-based flow cytometry has been developed in the late 1960's. While it has mostly been used with immuno-fluorescent staining and DNA marker dyes, with advent of fluorescent proteins, it has become a tool of choice for signal transduction studies in yeast. Its ability to provide quantitative measurement for a large number of cells within a relatively short amount of time allows to access single cell measurements with a high statistical significance ${ }^{28}$.

\subsection{Instrumentation}

To observe individual cells in suspension, the solution is aspirated at a low flow rate in the flow cytometer. A fast flow of so-called sheath fluid surrounds the medium to generate a thin core of sample (Figure 4). This hydrodynamic focusing technique allows to precisely position the flow of medium in the laser beam and present the cells one by one in front of an excitation source. Every time a cell crosses the laser path it will lead to scattering of the excitation light. The forward scattered light is detected by blocking the main laser beam and detecting the amount of light that passes around the obscuration bar. The amount of forward scattered light detected

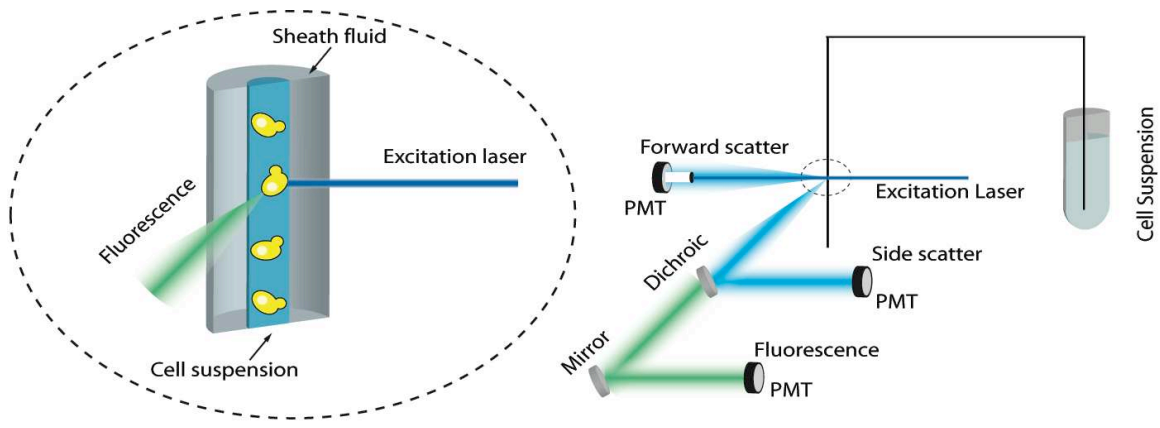

Figure 4. Schematic drawing of the flow cytometer detection system. Hydrodynamic focusing with the sheath fluid forces cells to pass one by one in front of the excitation laser. The resulting fluorescence and side-scatters are separated by appropriate optics and detected by photomultiplier tubes (PMT). The forward scatter is detected by blocking the excitation beam. 
scales with the volume of the cell. The light scattered at a $90^{\circ}$ (SideScattering) is also collected and provides an estimate of the granularity of the cell. Using the same excitation source, multiple fluorescence signals can be detected using appropriate filters to select the portion of spectrum of interest. The light is detected by photo-multiplier tubes, which have a high sensitivity and large dynamic range. For every scattering event, the fluorescence intensity is measured in all channels, therefore allowing to obtain for each individual cell a measurement of forward and side scatter along with multiple fluorescence measurements at various wavelengths. The rate of event detection is in the order of 1000 to $10^{\prime} 000$ cells per second, thus enabling to obtain in a short amount of time statically significant data sets.

Typical flow cytometers are equipped with a laser at $488 \mathrm{~nm}$ and multiple fluorescence detection channels around 530, 580 and $660 \mathrm{~nm}$ optimized for the detection of common synthetic dyes. Except for the GFP and YFP variants, which can both be excited at $488 \mathrm{~nm}$, this configuration is unfortunately not ideal for detecting combinations of fluorescent proteins. More complex set-up offer however multiple laser lines and a dozen of fluorescent detection channels which can be selected to excite CFP or RFP variants.

\subsection{Applications}

Flow cytometry is mostly used in conjunction with antibodies to label specific proteins. This technique is extremely successful in the analysis of the mammalian immune system to identify specific cell types or probe for the expression of surface receptors ${ }^{29,30}$. It has also been exploited to quantify cell cycle profiles. Using DNA intercalating fluorophores, it is straightforward to follow doubling of the DNA content thereby monitoring the progression of cells through the cell cycle ${ }^{31}$.

In yeast, since it is possible to tag a protein with GFP at its genomic locus, it becomes feasible to precisely quantify its expression level in single cells and study the variability of its abundance in a population. Given that the expression of a protein is mainly controlled by the promoter region upstream of the ORF ( 1000 base pairs before start codon), synthetic constructs can be generated based on a promoter driving the expression of a fluorescent protein. The promoter can be selected among the proteins that are known to be transcriptionally induced by a specific pathway ${ }^{32}$. Flow cytometric measurements can then be used to quantify the expression of this fluorescent reporter as function of stimulus to obtain a dose-response curve or the temporal evolution of the expression of this construct. 
As an example, we show in Figure 5A, the expression of a fluorescent reporter driven by the promoter of the pheromone-responsive gene (FIGl). Each sample is induced with $1 \mu \mathrm{M}$ of $\alpha$-factor and cycloheximide is added at different time points to block protein expression. After 1-2 hours of incubation to allow for protein maturation, ten thousand cells are measured for each sample. The sample taken before induction displays a basal level of fluorescence. This signal comes from the auto-fluorescence of the yeast and is certainly a limiting factor for the sensitivity of this method. Two hours of pheromone treatment leads to a 20 -fold increase in fluorescence intensity of the cells. Interestingly, 30 minutes after induction only a fraction of cells expressed the fluorescent reporter. This is due to the fact that only cells in the G1 phase of the cell cycle can induce the pathway ${ }^{33,34}$. The other cells will first need to complete cell division before becoming signaling competent (as shown by microscopy in Figure 5B). This explains the large extrinsic noise in the cellular response observed in the flow cytometry measurements.

One shortcoming of the flow cytometric measurements is that every cell can only be measured once. Therefore one cannot follow the fate of a given cell after pathway induction. This is somewhat compensated by the fact that the number of cells quantified in each measurement is so large that the sampling represents the behavior of all cells in the population. Another strategy, which can be used in certain situation, is the ability of the device to sort cells based on a set of measured parameters. For instance, it is possible
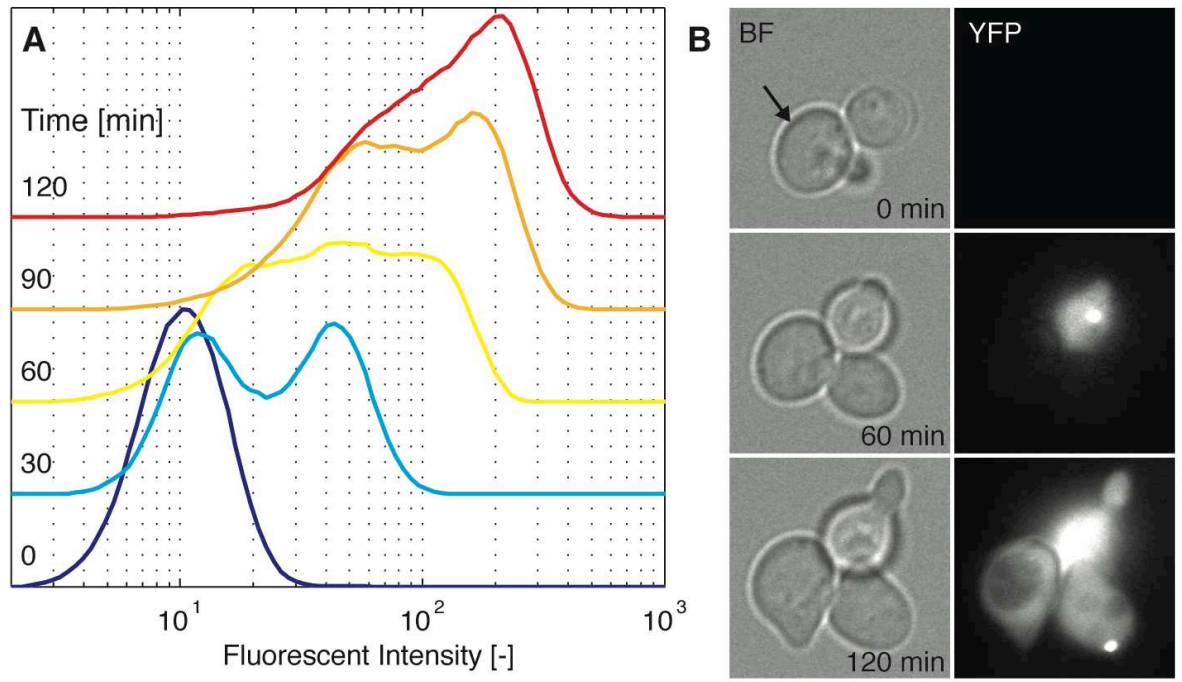

Figure 5. Temporal evolution of the expresssion of a fluorescent reporter ( $\mathrm{p} F I G 1$-YFP) upon activation of the mating pathway. (A) Flow cytometry. Cells were treated with cycloheximide and fluorescence was quantified after full maturation of the protein. (B) Live-cell microscopy. The budding cell (arrow) clearly show a delayed expression of the fluorescent reporter. 
to separate cells expressing and not-expressing a fluorescent reporter upon activation of the pathway. These cells can be re-cultured and stimulated a second time to verify if their behavior is inherited ${ }^{35}$.

\section{MICROSCOPY}

Microscopy has always been a tool of choice for biologists to study individual cell morphologies and phenotypes. With the recent advances in electronic detectors and image analysis software, this method is becoming highly quantitative and therefore can provide valuable data for mathematical modeling of biochemical pathways.

\subsection{Instrumentation}

Quantification of signaling pathways requires the measurements of the output by recording changes in the fluorescent signal. Many technical improvements have increased the sensitivity and reliability of microscopes in the last years. But it is probably equally important to properly control the stimulus applied to the cells. Given that microscopes offer the possibility to monitor cells over long periods of time, it also provides the opportunity to control the stimulus during the course of the experiment. Therefore researchers have employed flow channels and microfluidic devices to improve their understanding of the dynamics of signaling pathways.

\subsubsection{Microscopy samples and microfluidic devices}

To perform live imaging, cells are typically attached to the bottom of a well slide. The media present on top of the cells allows to sustain growth for many hours. If required, the microscope can be enclosed in an incubation chamber with temperature, humidity and $\mathrm{CO}_{2}$ control to provide the best conditions for cell growth. Stimulation of a signaling pathway can be achieved by adding soluble chemicals, such as sugars, drugs, pheromone or stress agents to the medium in the well. The cellular response induced by these compounds can then be observed microscopically in real time.

A limitation from this set-up is the inability to remove compounds from the medium. Therefore, for more refined experiments, flow chambers with coverslip bottoms have been developed (Figure 6B). This allows to keep a constant input of fresh medium and thereby offers the possibility to rapidly change the content of the medium allowing both addition and removal of stimuli to the cells. 
A particular type of flow chambers are microfluidic devices, which are made of 10 to $100 \mu \mathrm{m}$ channels molded in a polymer ${ }^{36,37}$. As shown in Figure $6 \mathrm{C}$, these devices are much more than a simple small dimension flow channel because they integrate all the element of the flow control and mixing within the chip. The small dimensions of the channels prevent turbulent mixing of flows and thereby allow the generation of complex temporal stimulation patterns such as pulses or ramps ${ }^{38,39}$. Due to the small volume present in these devices, the media switching times can be in the order of a few seconds. Using diffusion between two different media, it is also possible to generate stable concentration gradients for example to study the establishment of oriented cell polarity ${ }^{40}$. Another benefit of the small dimensions of microfluidic devices is the possibility to keep all cells in the same focal plane for multiple generations by designing chambers with a height of only a few microns ${ }^{41,42}$.

\subsubsection{Microscope light-path}

The light source in most epi-fluorescence microscopes is a mercury or metal-halide lamp. These lamps have a very broad emission covering the UV and visible part of the spectrum. An excitation filter is necessary to select the band of wavelength used for the excitation of the sample. Since the lamp is continuously emitting light it is also necessary to place a fast shutter in front of the lamp to precisely control the illumination time of the sample. High power light-emitting diodes (LED) are a new alternative for fluorescence imaging with the great advantage of being switched on electronically within a few microseconds. LEDs have well-defined emission spectra of 10 to 30 $\mathrm{nm}$ width, thus 4 to 6 units have to be coupled together to excite a wide range of fluorophores.

The filtered excitation light is reflected by a dichroic mirror and sent to the objective to excite the sample. The fluorescent light is emitted isotropically and only a small fraction is collected by the objective. It will pass through the dichroic mirror and is then filtered by an emission filter before being detected by the CCD camera (Figure 6A). The exposure time of the sample to the excitation light determines the brightness of the image. This parameter has to be selected carefully to obtain a good signal-to-noise ratio in the image while avoiding saturation of the high intensity pixels. For time-lapse imaging, it is also crucial to take into account the bleaching of the sample to avoid a decrease in the image quality at later time points. 
A

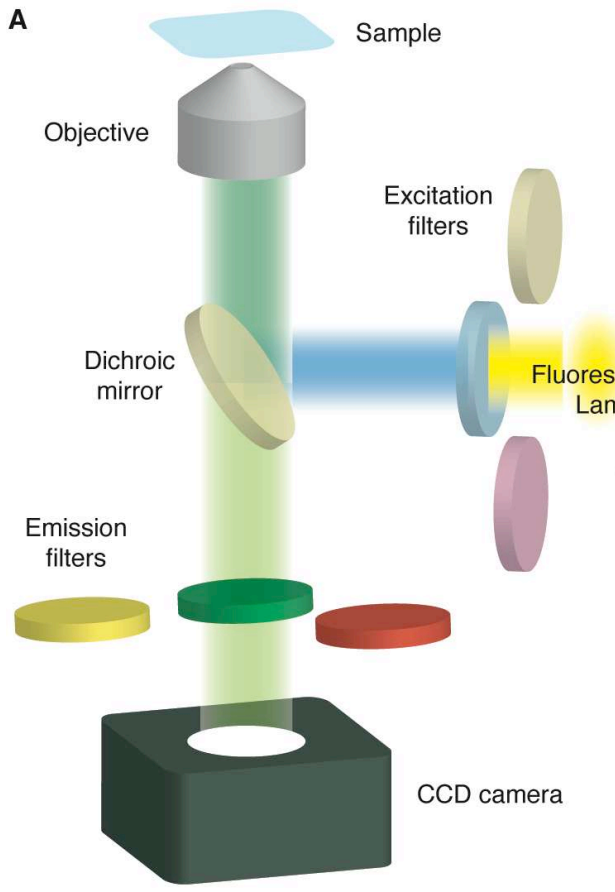

B

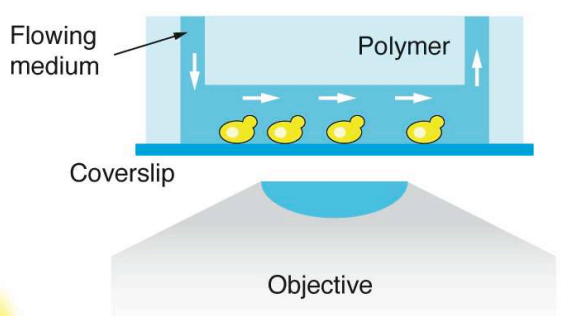

C

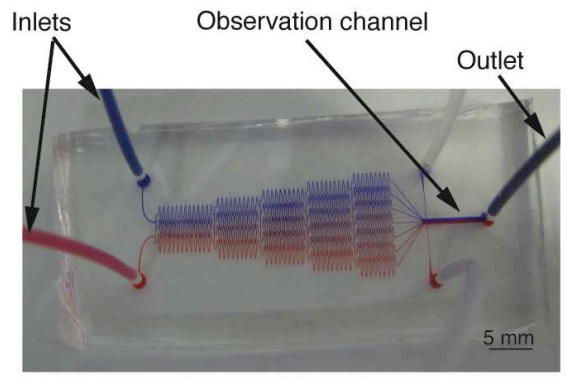

Figure 6. A. Light path in an epi-fluorescent microscope. The wide band emission of the fluorescence lamp is filtered and reflected by the dichroic mirror. The light is focused by the objective on the specimen. The emitted fluorescence is collected by the objective and is transmitted by the dichroic mirror. It is filtered by the emission filter before an image is recorded by the CCD camera. B. In a flow channel, cells are attached to the coverslip and the medium flows in the channels molded in the polymer. C. Picture of a gradient generating microfluidic device. The two inlet flows are mixed to generate a smooth concentration ramp in the observation channel.

The choice of the objective is always a trade-off between the size of the field of view and the optical resolution. For yeast cells, a 40x objective is usually ideal to quantify overall cellular fluorescence and allows to observe about 100 cells simultaneously. To observe cellular organelles, a higher magnification is necessary (60x or 100x) at the expense of fewer cells being imaged. To increase the statistics of the measurements, it is possible to record images at different locations in the sample. Microscopes are often equipped with highly reliable motorized XY-stages, which allow to repeatedly record multiple fields of view. The trade-off lies now between the number of stage positions to acquire and the dynamics of the process to be quantified. For very fast signaling events, this is clearly a limiting factor (1 min time interval can allow imaging of approx. 10 positions). 


\subsection{Image Analysis}

While flow cytometric assays directly provide a measure of the total cellular intensity, microscopy provides an image, which needs to be analyzed to extract the desired information. This is a complex task, which requires advanced image analysis algorithms. As shown in Figure 7, a microscopy image is in fact a 3D intensity map of pixel intensities. The thresholding process consists in finding the optimal intensity value to discriminate between background and object pixels. To identify the contour of individual cells, a water-shedding algorithm is often applied, which is based on the shape or intensity information from the segmented objects. Multiple fluorescent tags can be used to identify organelles and generate secondary objects within the cell. Once the final object shape has been obtained, it becomes relatively straightforward to extract the desired measurements. Geometrical measurements such as area, diameter or eccentricity can be extracted from the shape of the object. By quantifying the
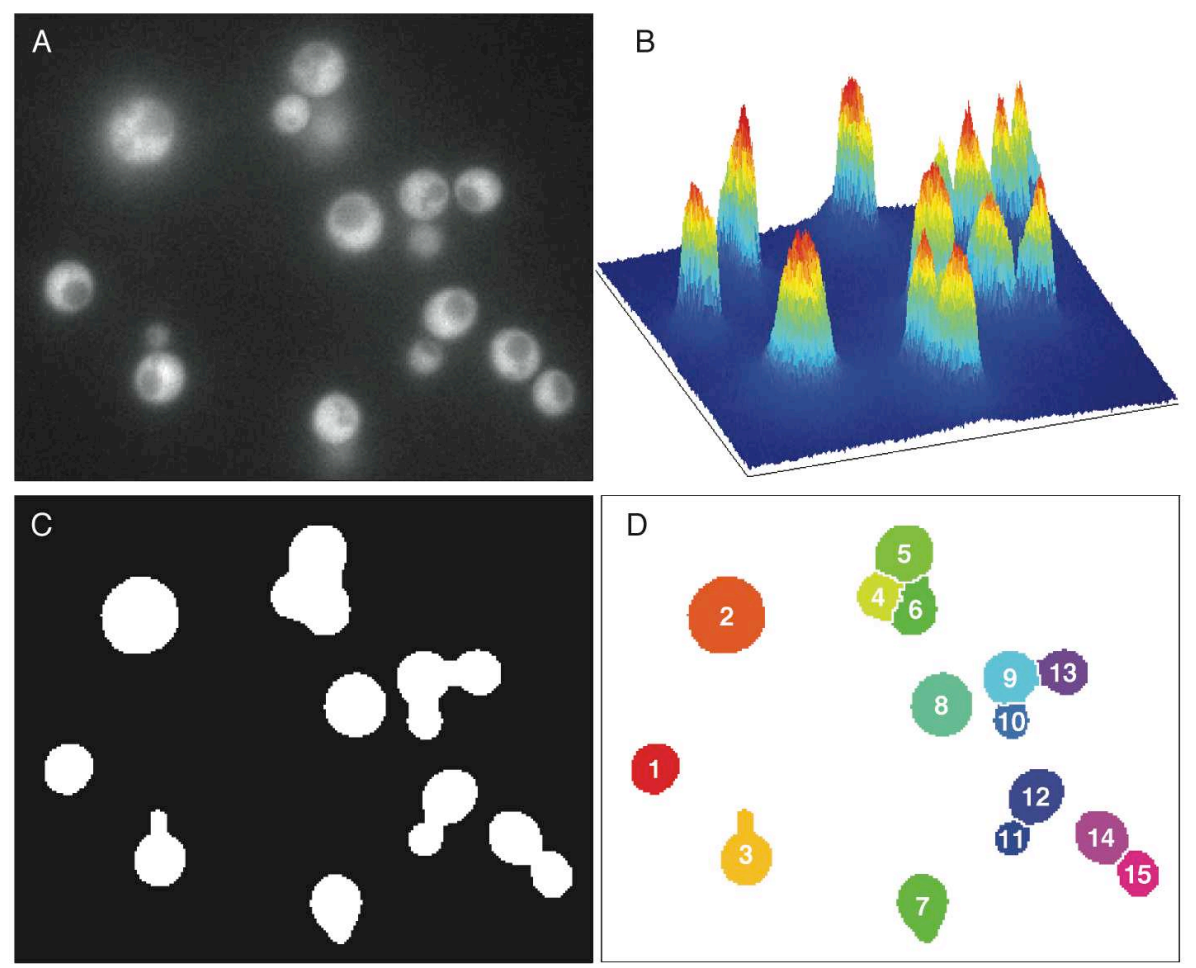

Figure 7. The original image (A) can be represented as 3D surface, where each pixel of the image has a given intensity (B). An intensity threshold is placed on the image to distinguish background and object pixels (C). The thresholded image is split between individual cells with a watershedding algorithm and each cell is labeled (D). 
intensity in each pixel of the object it is possible to obtain many more features such as the mean, maximum or total fluorescent intensity of the object.

Multiple software packages have been developed to analyze microscopy images ${ }^{43,44}$. In addition, ImageJ (http://rsb.info.nih.gov/ij/) and the image processing toolbox from Matlab (Mathworks) offer many low level routines that can be combined to generate a specific analysis workflow combining segmentation, objects recognition and feature measurements.

\subsection{Applications}

Using the same expression reporter described for the flow cytometry assays, it is possible to follow in real time the apparition of the fluorescence signal in a cell after inducing the signaling pathway. Unfortunately, due to the slow maturation of the protein, a roughly $30 \mathrm{~min}$. delay is observed between the expression of the protein and the apparition of the fluorescence (Figure 5B). The other issue with these types of reporters is the fact that the fluorescent proteins have a long lifetime in the cell. Observing the shutdown of a transcriptional process is therefore problematic because the cells remain fluorescent for many hours after protein production has been stopped.
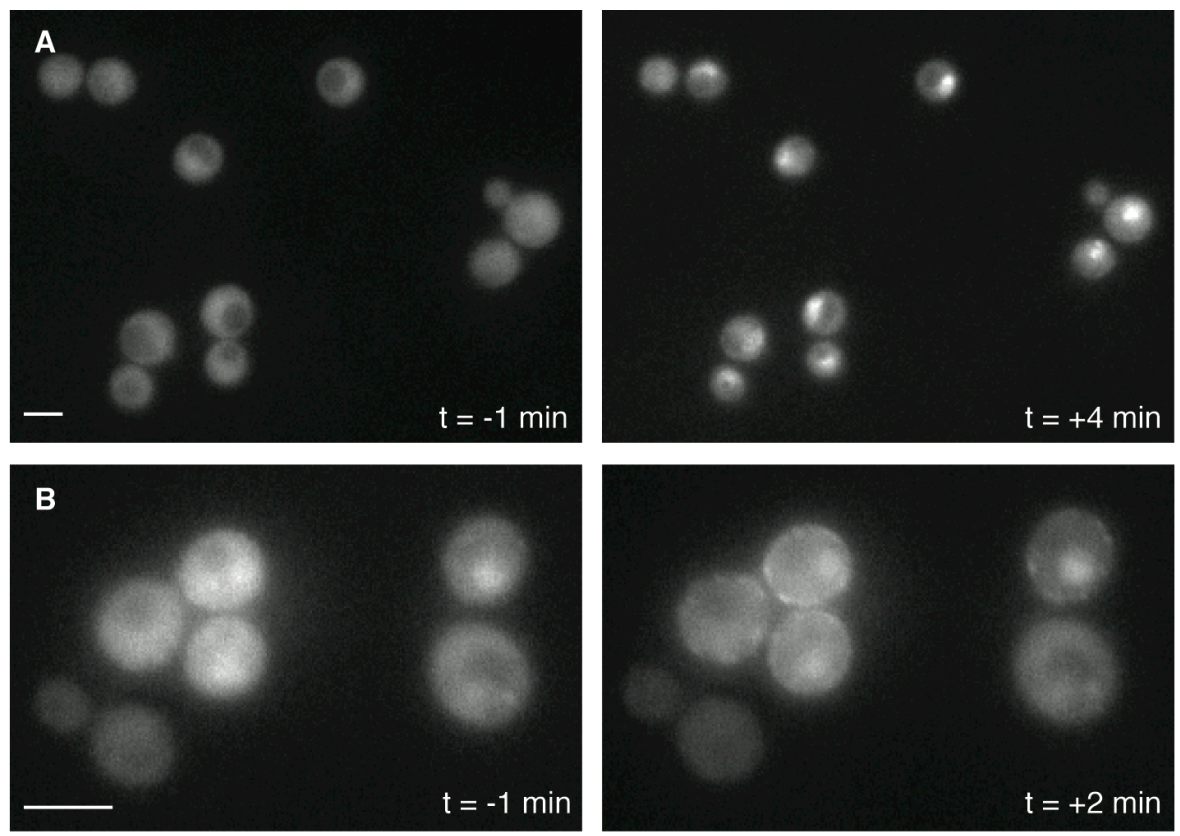

Figure 8. Quantification of signaling by relocation of active proteins. A. The MAPK Hog1p tagged with mCherry accumulates in the nucleus upon activation of the osmo-stress pathway with $\mathrm{NaCl}$. B. Relocation of the scaffod protein Ste5p to the plasma membrane upon treatment with mating pheromone. (scale bar $5 \mu \mathrm{m}$ ) 
To circumvent this problem, destabilized fluorescent protein have been generated which have a short lifetime ${ }^{45}$.

For more dynamic measurements of signaling cascade activation, it is sometimes possible to use a GFP-tagged protein that changes location upon stimulation of the cell. These types of experiments cannot be performed by flow cytomery because there is no net change of fluorescence. The subcellular resolution from the microscope is required to detect these events. Table 3 lists a few examples of relocation assays that can be used to quantify the activity of different signal transduction cascades.

One commonly used assay is the relocation of a protein to the nucleus of the cell. Many transcription factors or transcriptional regulators can display large variations in their nuclear concentration upon activation ${ }^{46}$. Figure $8 \mathrm{~A}$ illustrates the nuclear relocation of the Hog1p MAP kinase. Within a few minutes after osmotic stress, the change in fluorescence localization is apparent. This nuclear accumulation of the MAPK is closely linked to its activity state ${ }^{47}$ and therefore serves as a read-out for the activity of this pathway in single cells ${ }^{38,39}$.

In the mating pathway, activation of the pathway can be assessed by the membrane relocation of the scaffolding protein Ste $5 \mathrm{p}$. This assay was used to demonstrate the presence of a negative feedback loop from the MAPK, which tunes down the activation of the pathway after induction ${ }^{48}$. As shown in Figure 8B, Ste $5 p$ relocation can already be detected a few minutes after addition of pheromone to the cells. This clearly demonstrates the high temporal resolution, which can be obtained by these assays in contrast to the slow dynamics measured for fluorescent protein expression, which are further hindered by the delay due to maturation of the fluorophore.

Table 3. Examples of relocation assays for signal transduction quantification

\begin{tabular}{ccc}
\hline Relocation to & Stimulus & Protein \\
\hline Nucleus & General stress & Msn2 $\mathrm{p}^{49}$ \\
& Osmotic shock & Hog1 $\mathrm{p}^{47}$ \\
G1 cell cycle stage & Whi5 $\mathrm{p}^{45}$ \\
Pasma membrane & Mating pheromone & Ste5p $\mathrm{p}^{48}$ \\
Vacuolar membrane & Glucose levels & Vma5 $\mathrm{p}^{50}$ \\
Polarity landmark & Autophagy & Atg8 $\mathrm{p}^{51}$ \\
\hline
\end{tabular}


One general consideration when using reporters based on full-length proteins or endogenous promoters is that they often integrate multiple signals from various pathways. As an example, the Msn2p relocation is sensitive to a wide range of stresses such as starvation, osmotic shock or light-induced stress. The response to the stimulus can therefore be strongly influenced by the experimental conditions. In the case of Msn2p, after careful analysis of its phosphorylation pattern, a shorter version of the protein has been constructed to report specifically to glucose starvation and not to other stresses ${ }^{50}$. Engineering of synthetic reporter based on short regulated region of signaling proteins might offer more specificity and sensitivity to the assay.

\section{FUNCTIONAL MICROSCOPY}

Activation of a signaling cascade triggers the assembly (or disassembly) of protein complexes and the change in activity of proteins by modifications such as phosphorylation, acetylation or ubiquitination. Relocation and expression assays report on the integration of multiple elements of the signaling cascade. Functional imaging techniques such as Förster resonance energy transfer (FRET) and fluorescence correlation spectroscopy (FCS) techniques can monitor one single interaction or modification in a signaling cascade.

\subsection{Förster Resonance Energy Transfer}

Förster Resonance Energy Transfer is defined as the transfer of excitation energy of a donor to an acceptor fluorophore via dipole-dipole interaction. Due the nature of this interaction, the efficiency of the transfer $(E)$ decreases with the sixth power of the distance between the two dyes $\mathrm{R}^{53,54}$.

$$
\mathrm{E}=1-\frac{\mathrm{I}_{\mathrm{F}}}{\mathrm{I}_{\mathrm{D}}}=\frac{\mathrm{R}_{0}^{6}}{\mathrm{R}_{0}^{6}+\mathrm{R}^{6}}
$$

Where $I_{F}$ and $I_{D}$ are respectively the fluorescent intensities of the donor in presence and absence of FRET. The Förster distance $\mathrm{R}_{0}$ corresponds to the separation where $50 \%$ of the donor fluorescence is converted into the energy transfer process.

$$
\mathrm{R}_{0}^{6}=\frac{9000 \ln (10) \kappa^{2} \mathrm{Q}_{\mathrm{D}}}{128 \pi^{5} \mathrm{~N}_{\mathrm{A}} \mathrm{n}^{4}} \mathrm{~J}
$$


This parameter depends on the index of refraction of the medium, $\mathrm{n}$; Avogadro's number, $\mathrm{N}_{\mathrm{A}}$; the fluorescence quantum yield of the donor molecule, $\mathrm{Q}_{\mathrm{D}}$; the orientation factor between the two dipoles, $\kappa$ and the overlap integral, J. This integral scales with the overlap of the emission spectrum of the donor and the absorption spectrum of the acceptor. For a well-matched fluorophore pair, $\mathrm{R}_{0}$ is on the order of 50-60 $\AA^{55,56}$.

Due to the high sensitivity of the FRET process with distance it is possible to probe the association of two proteins in vivo by tagging them with a set of fluorescent protein (CFP and YFP or GFP and mCherry). Indeed, unless these two proteins belong to the same complex, it is extremely unlikely for them to come by chance close enough to generate a FRET signal (Figure 9A).

This technique has been used to study the activation of the mating pathway at two different points along the signal transduction cascade. A loss of FRET was observed upon dissociation of the hetero-trimeric G-protein in $\alpha$-factor treated cells ${ }^{48,57}$. Moreover, dissociation of the repressor Dig $1 \mathrm{p}$ from the transcription factor Ste12p has also been probed by FRET. Since this dissociation is triggered by phosphorylation of Ste12p and Dig1p by Fus $3 p$, the loss of FRET reports faithfully on the nuclear activity of the MAPK ${ }^{48}$.

The FRET efficiency is commonly quantified by measuring the acceptor sensitization (i.e. the additional fluorescence generated by the FRET process
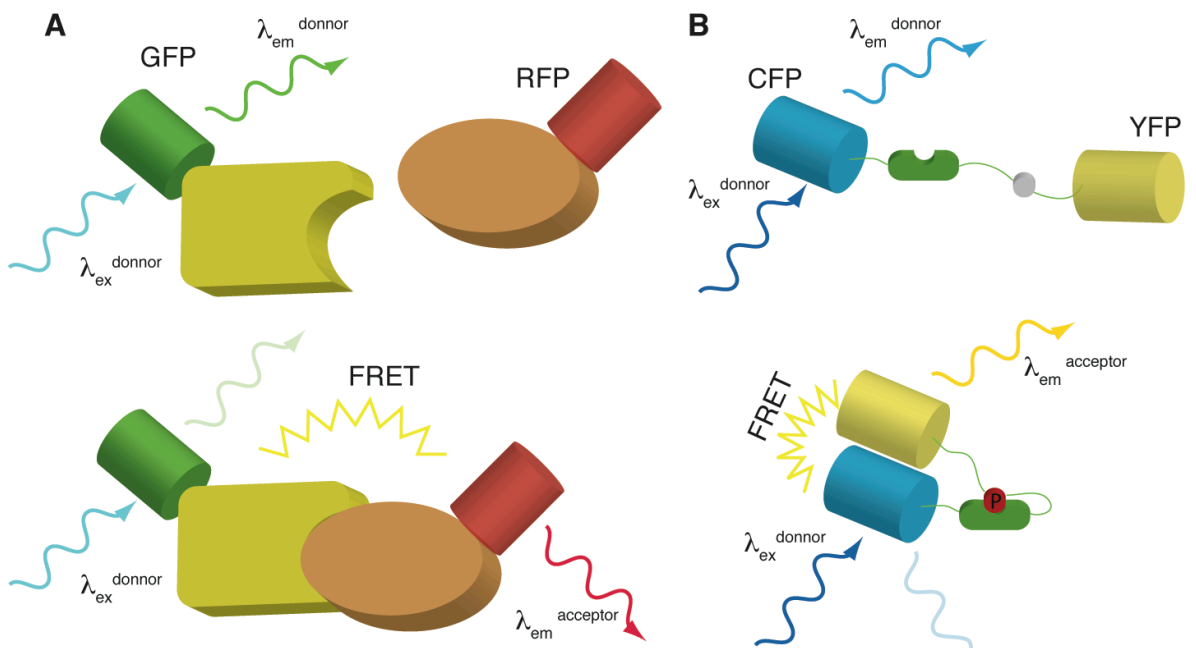

B

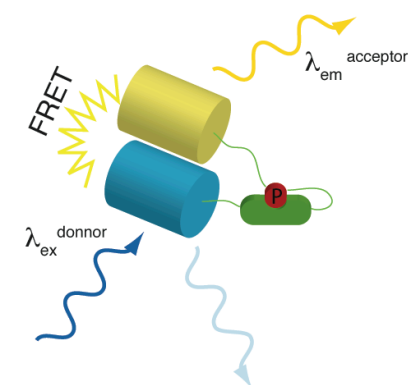

Figure 9. Two different strategies to make use of the FRET process in vivo to quantify signal transduction. A. Protein-protein interraction: upon binding of the two fluorescently-tagged proteins, the two flluorescent protein variants come in close proximity and energy transfer can occur. B. FRET sensor: a synthetic construct made of two fluorescent proteins separated by a functional linker which will undergo a large conformation change upon modification or ligand binding. 
in the acceptor channel upon donor excitation). For FRET measurements, it is necessary to acquire at least three images for the donor, the acceptor and the emission of the acceptor upon donor excitation ${ }^{58}$.

Although appealing, the application of this technique is challenging. Indeed, the size of the interacting proteins and of the fluorescent tags can lead to a large distance and unfavorable orientation between the chromophores, which can result in an undetectable FRET signal. Moreover, due to the variation in the level of expression of the two proteins, it is hard to decouple the change in FRET efficiency from the changes in the intensities of the donor and acceptors. Finally, the FRET efficiency measures a combination of the amount of energy transfer and the number of complexes formed. The same efficiency value can be measured from a sample with a high transfer efficiency and only few complexes present or a sample with low energy transfer but with all donors engaged in complexes.

Only the measurement of the fluorescence lifetime of the donor can decouple the efficiency of the FRET process, the amount of complexes formed and the concentration of donor present ${ }^{59}$.

$$
\mathrm{E}=1-\frac{\tau_{\mathrm{F}}}{\tau_{\mathrm{D}}}
$$

The deactivation of the donor excited-state by FRET shortens the lifetime of the excited state of the dye $\left(\tau_{\mathrm{F}}\right)$ compared to a non-fretting donor $\left(\tau_{\mathrm{D}}\right)$. This technique requires a complex acquisition set-up to record the lifetime of the fluorescence in every pixel of the image ${ }^{60,61}$. This method was used to detect a gradient of MAPK activity arising from the tip of the mating projection. The authors quantified by fluorescence lifetime imaging the FRET between a GFP-tagged Fus3p and a Cy3-labeled antibody specific for the active form of the MAPK ${ }^{62}$.

Another way to use Förster resonance energy transfer for signaling studies is to develop FRET sensors. These sensors consist of pair of fluorescent proteins linked by a short peptide. Due to the high sensitivity of the FRET efficiency to chromophore distance and orientation, a change in the conformation of the linking peptide can lead to a strong change in the FRET signal (Figure 9B). This strategy has been used to sense intracellular metabolites by engineering a binding site in the linker region ${ }^{63,64}$. It is also possible to probe kinase activity by combining in the FRET sensor a phospho-acceptor site for a specific kinase and a sequence binding to this phophorylated residue ${ }^{65,66}$. This can offer a direct read-out for the activity of a protein upon stimulation of the pathway. The great advantage of this technique is that the two chromophores are linked together and therefore are always expressed at the same level. Many of the problems involved in 
quantification of the FRET process for protein interactions are thus circumvented. The FRET efficiency can be simply calculated from the ratio between two images acquired with donor excitation and emission filters for the donor or the acceptor.

\subsection{Fluorescence Correlation Spectroscopy}

In contrast to FRET, Fluorescence correlation spectroscopy (FCS) offers the possibility to study protein complex formation quantitatively and with a high sensitivity independently of distance or orientation ${ }^{67,68,69}$. This technique relies on the measurement of the fluctuations in the fluorescence signal generated by single molecules moving through the confocal volume of a microscope. This volume is on the order of $10^{-14}$ liter and contains on average 10 molecules for a concentration of approx. $10 \mathrm{nM}$. If one of these molecules leaves the observation volume, a drop of $10 \%$ in the fluorescent signal should be detected. Based on Poisson statistics, if the average number of molecule is 10 , the standard deviation is the square root of 10 , and we therefore expect a variation in the signal of roughly $30 \%$ over time. The rate at which those fluctuations happen is directly related to the diffusion coefficient of the molecule in the medium and therefore the residence time of each molecule in the confocal volume (Figure 10).

To analyze these fluctuations an auto-correlation of the fluorescence signal as function of time is calculated. The auto-correlation curve $\mathrm{G}(\tau)$ will obey to the following equation for a simple diffusion model:

$$
\mathrm{G}(\tau)=\frac{\mathrm{G}_{0}}{\langle\mathrm{~N}\rangle} \frac{1}{1+\frac{\tau}{\tau_{\mathrm{D}}}} \frac{1}{\sqrt{1+\frac{\mathrm{r}_{0}^{2}}{\mathrm{Z}^{2}} \frac{\tau}{\tau_{\mathrm{D}}}}}
$$

The brightness factor $\mathrm{G}_{0}$ as well as the lateral $\mathrm{r}_{0}$ and axial $\mathrm{Z}$ dimensions of the confocal volume are parameters that have to be determined experimentally with a reference sample. Interestingly, the amplitude of the correlation function scales with the inverse the average number of molecules in the volume $\langle\mathrm{N}\rangle$. This follows naturally from the fact that relative changes in intensity, when one molecule leaves the confocal volume, will be smaller as the concentration increases. Therefore, the sensitivity of FCS is maximized at low concentrations. The other parameter, which can be extracted from the fitting of the auto-correlation function, is the diffusion time of the dye $\left(\tau_{\mathrm{D}}\right)$. This parameter could be used to measure the binding of a fluorescently-tagged protein to a complex. Unfortunately, since the diffusion constant scales approximately with the cubic root of the mass, the 
change in size between the free and complex-bound protein needs to be very large to be able to quantify it. It is however possible to probe homo-dimer formation due to a change in brightness of the particles ${ }^{70}$.

Studying the interaction of two proteins can be achieved by tagging both of them with different fluorescent protein variants (typically GFP and mCherry). Under these conditions, one can record the fluctuations in both color channels in parallel and calculate a cross-correlation curve ${ }^{71,72}$. If the two proteins of interest are present in the same complex, they will generate similar fluctuations in both detection channels, which will result in an increased cross-correlation signal. This technique allows to obtain the absolute concentration of the proteins along with the ratio of proteins engaged in the complex and therefore enables the characterization of the affinity constant of the two proteins. This method has been applied successfully in vitro, but experimental artifacts such as autofluorescence have slowed down its adoption for live cell assays.

Two groups investigated complex formation in the mating pathway by $\mathrm{FCS}^{62,73}$. Maeder et al. studied the pair-wise interactions between Ste11p, Ste $7 p$, Ste $5 p$ and Fus $3 p$ in the cytoplasm of $\alpha$-factor treated and untreated cells. They quantified the cytoplasmic concentration of each proteins and by

A

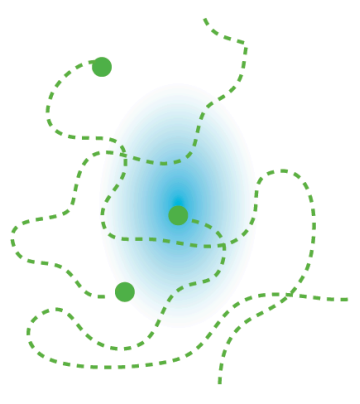

B

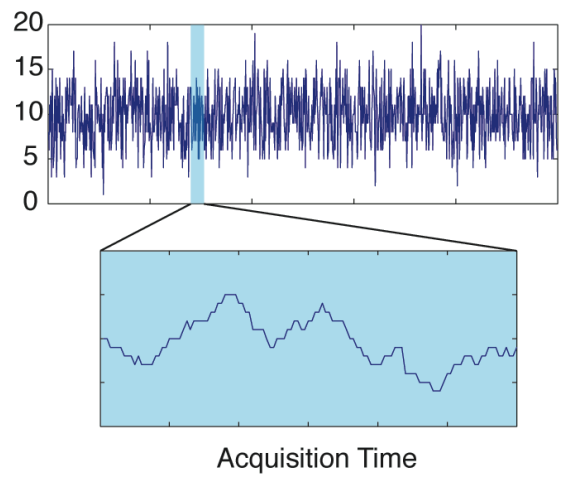

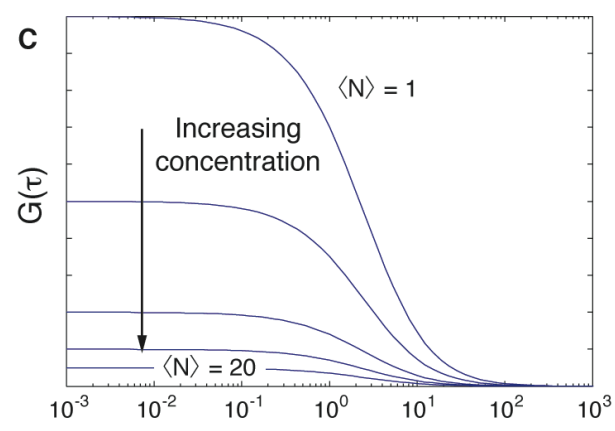

D

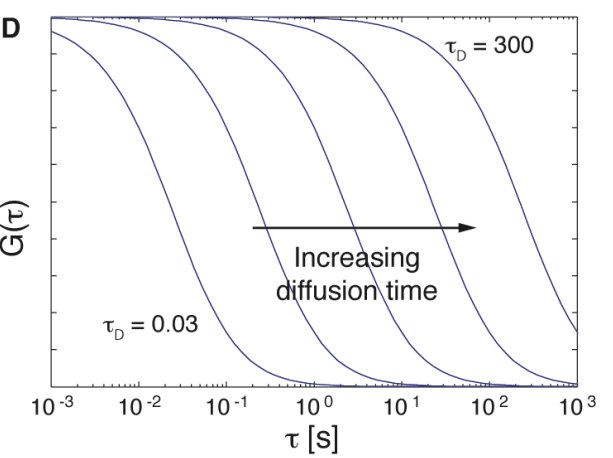

Figure 10. Random diffusion of fluorescent particules through the confocal volume of a microscope (A) generates a fluctuating fluorescence signal (B). Autocorrelation of these fluctuations allows the determination of the concentration (C) and the diffusion time (D) of the fluorescently-tagged particle. 
cross-correlation measurements the concentration of the complexes between the two proteins. Their measurements lead them to conclude that there is no change in the cytoplasmic complex formation upon $\alpha$-factor treatment for any of the complexes investigated. In contrast to this, Slaughter et al. ${ }^{73}$ performing similar measurements found a regulated interaction for Fus $3 p$ and Ste $7 p$ upon $\alpha$-factor stimulation. They could not detect an interaction between the two proteins in cycling cells but measured the formation of a complex upon $\alpha$-factor stimulation. Both studies however agree on the fact that most of the change in protein interactions happen at the cell membrane and particularly at the tip of the mating projection were these proteins accumulate. Unfortunately, it is difficult to probe this region by FCS since the proteins will have an increased residence time due to their involvement in membrane bound complexes and therefore will not give rise to fluctuations.

\section{OUTLOOK}

Flow cytometry and quantitative microscopy significantly improved our understanding of signal transduction in single cells. While flow cytometry allows to rapidly quantify the total fluorescence of a large number of cells, microscopy allows to follow in real time the dynamics of signal transduction in the same cell by monitoring the relocation or expression of reporter proteins. Using FRET or FCS, it becomes possible to track directly the changes in activity or binding affinity of key signaling molecules.

The pace of the technical development of microscopy and flow cytometry will keep increasing in the coming years and will blend the boundary between these two complementary techniques. The cytometer, which offers excellent statistics, delivers poor information content for each cell. A newer generation of instruments provide the capability of imaging the cells in the flow $^{74,75}$. This allows to extract more complex features from each cell and will for instance allow to study a nuclear relocation event with high temporal resolution and improved statistics.

On the other side, genome-wide screens have been performed on microscopes, which resulted in the development of high-throughput acquisition and image analysis techniques ${ }^{76}$. These screens, which involved the measurements of millions of cells, were mostly performed for endpoint measurements. Signaling studies with their special needs for high temporal resolution are less prone to such approaches, but a combination of microfluidics and microscopy could solve this issue ${ }^{77}$. 
The major challenge will remain the development of sensitive and high fidelity sensors for the measurements of signal transduction. We have presented in this chapter a number of tools used to monitor the activation of signaling cascades such as expression reporters, relocation assays or FRET sensors. It is now possible to combine several of these sensors in the same cell to correlate the activity of the pathway at multiple levels in single cells.

\section{ACKNOWLEDGEMENTS}

We would like to thank Reinhard Dechant for critical reading of the manuscript. Work in the laboratory of M.P. is supported by Unicellsys, SPMD, the SystemsX.ch initiative (YeastX and LiverX projects), the ETHZ and the Swiss National Science Foundation (SNF).

\section{REFERENCES}

Ferrell, J. E. \& Machleder, E. M. The biochemical basis of an all-or-none cell fate switch in Xenopus oocytes. Science 280, 895-898, (1998).

2 Muzzey, D. \& van Oudenaarden, A. Quantitative time-lapse fluorescence microscopy in single cells. Annu Rev Cell Dev Biol 25, 301-327, (2009).

3 Santos, S. D. M., Verveer, P. J. \& Bastiaens, P. I. H. Growth factor-induced MAPK network topology shapes Erk response determining PC-12 cell fate. Nat Cell Biol 9, 324-330, (2007).

4 Cai, L., Dalal, C. K. \& Elowitz, M. B. Frequency-modulated nuclear localization bursts coordinate gene regulation. Nature 455, 485-490, (2008).

5 Garmendia-Torres, C., Goldbeter, A. \& Jacquet, M. Nucleocytoplasmic oscillations of the yeast transcription factor Msn2: evidence for periodic PKA activation. Curr Biol 17, 1044-1049, (2007).

6 KAErn, M., Elston, T. C., Blake, W. J. \& Collins, J. J. Stochasticity in gene expression: from theories to phenotypes. Nat Rev Genet 6, 451-464, (2005).

7 Elowitz, M. B., Levine, A. J., Siggia, E. D. \& Swain, P. S. Stochastic gene expression in a single cell. Science 297, 1183-1186, (2002).

8 Taniguchi, Y. et al. Quantifying E. coli proteome and transcriptome with singlemolecule sensitivity in single cells. Science 329, 533-538, (2010).

9 Colman-Lerner, A. et al. Regulated cell-to-cell variation in a cell-fate decision system. Nature 437, 699-706, (2005).

10 Amantonico, A., Oh, J. Y., Sobek, J., Heinemann, M. \& Zenobi, R. Mass spectrometric method for analyzing metabolites in yeast with single cell sensitivity. Angew Chem Int Ed Engl 47, 5382-5385, (2008).

11 Monroe, E., Jurchen, J., Rubakhin, S. \& Sweedler, J. Single-cell measurements with mass spectrometry. New frontiers in ultrasensitive bioanalysis: advanced analytical 
chemistry applications in nanobiotechnology, single molecule detection, and single cell analysis, 269, (2007).

12 Elf, J., Li, G.-W. \& Xie, X. S. Probing transcription factor dynamics at the singlemolecule level in a living cell. Science 316, 1191-1194, (2007).

13 Xie, X. S., Choi, P. J., Li, G.-W., Lee, N. K. \& Lia, G. Single-molecule approach to molecular biology in living bacterial cells. Annual review of biophysics 37, 417444, (2008).

14 Tsien, R. Y. The green fluorescent protein. Annu Rev Biochem 67, 509-544, (1998).

15 Zimmer, M. Green Fluorescent Protein (GFP): Applications, structure, and related photophysical behavior. Chem. Rev. 102, 759-781, (2002).

16 Huh, W.-K. et al. Global analysis of protein localization in budding yeast. Nature 425, 686-691, (2003).

17 Shaner, N. C., Steinbach, P. A. \& Tsien, R. Y. A guide to choosing fluorescent proteins. Nature Methods 2, 905-909, (2005).

18 Miesenböck, G., De Angelis, D. A. \& Rothman, J. E. Visualizing secretion and synaptic transmission with $\mathrm{pH}$-sensitive green fluorescent proteins. Nature 394, 192-195, (1998).

19 van Drogen, F. \& Peter, M. Revealing protein dynamics by photobleaching techniques. Methods Mol Biol 284, 287-306, (2004).

20 van Drogen, F., Stucke, V. M., Jorritsma, G. \& Peter, M. MAP kinase dynamics in response to pheromones in budding yeast. Nat Cell Biol 3, 1051-1059, (2001).

21 Patterson, G. H. \& Lippincott-Schwartz, J. Selective photolabeling of proteins using photoactivatable GFP. Methods 32, 445-450, (2004).

22 Ando, R., Hama, H., Yamamoto-Hino, M., Mizuno, H. \& Miyawaki, A. An optical marker based on the UV-induced green-to-red photoconversion of a fluorescent protein. Proc Natl Acad Sci USA 99, 12651-12656, (2002).

23 Goffeau, A. et al. Life with 6000 genes. Science 274, 546, 563-547, (1996).

24 Chen, R. E. \& Thorner, J. Function and regulation in MAPK signaling pathways: lessons learned from the yeast Saccharomyces cerevisiae. Biochim Biophys Acta 1773, 1311-1340, (2007).

25 Bardwell, L. A walk-through of the yeast mating pheromone response pathway. Peptides 26, 339-350, (2005).

26 Rudolf, F., Pelet, S. \& Peter, M. Regulation of MAPK signaling in yeast. Topics in Current Genetics 20, 187-204, (2007).

27 Lamson, R., Takahashi, S., Winters, M. \& Pryciak, P. M. Dual Role for Membrane Localization in Yeast MAP Kinase Cascade Activation and Its Contribution to Signaling Fidelity. Current Biology 16, 618-623, (2006).

28 Nolan, J. P. \& Yang, L. The flow of cytometry into systems biology. Brief Funct Genomic Proteomic 6, 81-90, (2007).

29 Drouet, M. \& Lees, O. Clinical applications of flow cytometry in hematology and immunology. Biol Cell 78, 73-78, (1993).

30 Shapiro, H. M. Multistation multiparameter flow cytometry: a critical review and rationale. Cytometry 3, 227-243, (1983).

31 Rieseberg, M., Kasper, C., Reardon, K. F. \& Scheper, T. Flow cytometry in biotechnology. Appl Microbiol Biotechnol 56, 350-360, (2001). 
32 Gasch, A. P. et al. Genomic expression programs in the response of yeast cells to environmental changes. Mol Biol Cell 11, 4241-4257, (2000).

33 Oehlen, L. J. \& Cross, F. R. G1 cyclins CLN1 and CLN2 repress the mating factor response pathway at Start in the yeast cell cycle. Genes \& Development 8, 10581070, (1994).

34 Strickfaden, S. C. et al. A mechanism for cell-cycle regulation of MAP kinase signaling in a yeast differentiation pathway. Cell 128, 519-531, (2007).

35 Acar, M., Becskei, A. \& van Oudenaarden, A. Enhancement of cellular memory by reducing stochastic transitions. Nature 435, 228-232, (2005).

36 Whitesides, G. M. The origins and the future of microfluidics. Nature 442, 368-373, (2006).

37 Bennett, M. R. \& Hasty, J. Microfluidic devices for measuring gene network dynamics in single cells. Nat Rev Genet 10, 628-638, (2009).

38 Hersen, P., McClean, M. N., Mahadevan, L. \& Ramanathan, S. Signal processing by the HOG MAP kinase pathway. Proc Natl Acad Sci USA 105, 7165-7170, (2008).

39 Muzzey, D., Gómez-Uribe, C. A., Mettetal, J. T. \& van Oudenaarden, A. A systems-level analysis of perfect adaptation in yeast osmoregulation. Cell 138, 160171, (2009).

40 Paliwal, S. et al. MAPK-mediated bimodal gene expression and adaptive gradient sensing in yeast. Nature 446, 46-51, (2007).

41 Charvin, G., Cross, F. R. \& Siggia, E. D. A microfluidic device for temporally controlled gene expression and long-term fluorescent imaging in unperturbed dividing yeast cells. PLoS ONE 3, e1468, (2008).

42 Lee, P. J., Helman, N. C., Lim, W. A. \& Hung, P. J. A microfluidic system for dynamic yeast cell imaging. BioTechniques 44, 91-95, (2008).

43 Carpenter, A. E. et al. CellProfiler: image analysis software for identifying and quantifying cell phenotypes. Genome Biol 7, R100, (2006).

44 Gordon, A. et al. Single-cell quantification of molecules and rates using opensource microscope-based cytometry. Nat Methods 4, 175-181, (2007).

45 Bean, J. M., Siggia, E. D. \& Cross, F. R. Coherence and timing of cell cycle start examined at single-cell resolution. Mol Cell 21, 3-14, (2006).

46 Cyert, M. S. Regulation of nuclear localization during signaling. J Biol Chem 276, 20805-20808, (2001).

47 Reiser, V., Ruis, H. \& Ammerer, G. Kinase activity-dependent nuclear export opposes stress-induced nuclear accumulation and retention of Hog1 mitogenactivated protein kinase in the budding yeast Saccharomyces cerevisiae. Mol Biol Cell 10, 1147-1161, (1999).

$48 \mathrm{Yu}, \mathrm{R}$. C. et al. Negative feedback that improves information transmission in yeast signalling. Nature 456, 755-761, (2008).

49 Görner, W. et al. Nuclear localization of the $\mathrm{C} 2 \mathrm{H} 2$ zinc finger protein $\mathrm{Msn} 2 \mathrm{p}$ is regulated by stress and protein kinase A activity. Genes \& Development 12, 586597, (1998).

50 Dechant, R. et al. Cytosolic $\mathrm{pH}$ is a second messenger for glucose and regulates the PKA pathway through V-ATPase. EMBO J, (2010). 
51 Kraft, C., Deplazes, A., Sohrmann, M. \& Peter, M. Mature ribosomes are selectively degraded upon starvation by an autophagy pathway requiring the Ubp3p/Bre5p ubiquitin protease. Nat Cell Biol 10, 602-610, (2008).

52 Shimada, Y., Wiget, P., Gulli, M.-P., Bi, E. \& Peter, M. The nucleotide exchange factor Cdc24p may be regulated by auto-inhibition. EMBO J 23, 1051-1062, (2004).

53 Förster, T. Zwischenmolekulare Energiewanderung und Fluoreszenz. Ann. Phys. 2, 55-75, (1948).

54 Jares-Erijman, E. A. \& Jovin, T. M. FRET imaging. Nat Biotechnol 21, 1387-1395, (2003).

55 Patterson, G. H., Piston, D. W. \& Barisas, B. G. Förster distances between green fluorescent protein pairs. Anal. Biochem. 284, 438-440, (2000).

56 Wu, P. \& Brand, L. Resonance energy transfer: methods and applications. Anal Biochem 218, 1-13, (1994).

57 Yi, T.-M., Kitano, H. \& Simon, M. I. A quantitative characterization of the yeast heterotrimeric G protein cycle. Proc Natl Acad Sci USA 100, 10764-10769, (2003).

58 Berney, C. \& Danuser, G. FRET or no FRET: A quantitative study. Biophys. J. 84, 3992-4010, (2003).

59 Pelet, S., Previte, M. J. R. \& So, P. T. C. Comparing the quantification of Forster resonance energy transfer measurement accuracies based on intensity, spectral, and lifetime imaging. Journal of biomedical optics 11, 34017, (2006).

60 Becker, W. et al. Fluorescence lifetime imaging by time-correlated single-photon counting. Microsc. Res. Techniq. 63, 58 - 66, (2004).

61 Verveer, P. J., Squire, A. \& Bastiaens, P. I. H. in Methods in cellular imaging (ed A. Periasamy) 273-294 (Oxford University Press, 2001).

62 Maeder, C. I. et al. Spatial regulation of Fus3 MAP kinase activity through a reaction-diffusion mechanism in yeast pheromone signalling. Nat Cell Biol 9, 13191326, (2007).

63 Fehr, M., Ehrhardt, D. W., Lalonde, S. \& Frommer, W. B. Minimally invasive dynamic imaging of ions and metabolites in living cells. Curr Opin Plant Biol 7, 345-351, (2004).

64 Fehr, M., Frommer, W. B. \& Lalonde, S. Visualization of maltose uptake in living yeast cells by fluorescent nanosensors. Proc Natl Acad Sci USA 99, 9846-9851, (2002).

65 Ting, A. Y., Kain, K. H., Klemke, R. L. \& Tsien, R. Y. Genetically encoded fluorescent reporters of protein tyrosine kinase activities in living cells. Proc Natl Acad Sci USA 98, 15003-15008, (2001).

66 Harvey, C. D. et al. A genetically encoded fluorescent sensor of ERK activity. Proc Natl Acad Sci USA 105, 19264-19269, (2008).

67 Magde, D., Elson, E. \& Webb, W. Thermodynamic fluctuations in a reacting system-measurement by fluorescence correlation spectroscopy. Phys. Rev. Lett. 29, 705-708, (1972).

68 Schwille, P., Haupts, U., Maiti, S. \& Webb, W. W. Molecular dynamics in living cells observed by fluorescence correlation spectroscopy with one- and two-photon excitation. Biophys J 77, 2251-2265, (1999). 
69 Schwille, P. Fluorescence correlation spectroscopy and its potential for intracellular applications. Cell Biochem Biophys 34, 383-408, (2001).

70 Chen, Y., Müller, J. D., So, P. T. \& Gratton, E. The photon counting histogram in fluorescence fluctuation spectroscopy. Biophys $J$ 77, 553-567, (1999).

71 Hwang, L. C. \& Wohland, T. Recent advances in fluorescence cross-correlation spectroscopy. Cell Biochem Biophys 49, 1-13, (2007).

72 Schwille, P., Meyer-Almes, F. J. \& Rigler, R. Dual-color fluorescence crosscorrelation spectroscopy for multicomponent diffusional analysis in solution. Biophys J 72, 1878-1886, (1997).

73 Slaughter, B. D., Schwartz, J. W. \& Li, R. Mapping dynamic protein interactions in MAP kinase signaling using live-cell fluorescence fluctuation spectroscopy and imaging. Proc Natl Acad Sci USA 104, 20320-20325, (2007).

74 George, T. C. et al. Distinguishing modes of cell death using the ImageStream multispectral imaging flow cytometer. Cytometry A 59, 237-245, (2004).

75 George, T. C. et al. Quantitative measurement of nuclear translocation events using similarity analysis of multispectral cellular images obtained in flow. J Immunol Methods 311, 117-129, (2006).

76 Pepperkok, R. \& Ellenberg, J. High-throughput fluorescence microscopy for systems biology. Nat Rev Mol Cell Biol 7, 690-696, (2006).

77 Taylor, R. et al. Dynamic analysis of MAPK signaling using a high-throughput microfluidic single-cell imaging platform. Proc Natl Acad Sci USA, (2009). 